\title{
A Prospective study of the National Early Warning Score in Cardiothoracic Surgery
}

\author{
M Tarazi", N Chan, N Mayooran, R Neagu, B Philip, MN Anjum, K Doddakula \\ From World Society of Cardiothoracic Surgeons 25th Anniversary Congress, Edinburgh \\ Edinburgh, UK. 19-22 September 2015
}

\section{Background/Introduction}

The National Early Warning Score (NEWS) is a clinical guide used to facilitate early detection of deterioration by categorising a patient's severity of illness and prompting nursing staff to request a medical review at specific trigger points utilising a structured communication tool while following a definitive escalation plan. Adopting a National Early Warning Score (NEWS) is beneficial for standardising the assessment of acute illness severity, enabling a more timely response using a common language across acute hospitals nationally.

\section{Aims/Objectives}

To determine whether the NEWS guideline recommendations apply to post-operative cardiac and thoracic patients.

\section{Method}

A prospective study of patients undergoing cardiac and thoracic surgery was performed. Data was entered into a spread sheet using patient's medical notes and observation sheets. The data looked at procedure performed, post-operative length of stay in ICU, post-operative complications, NEWS score on arrival to ward, and average NEWS score each day up to and including day of discharge.

\section{Results}

100 post-operative cardiac patients and 100 post-operative thoracic patients were included in the study. Our results showed initially post-operative cardiac and thoracic patients had a high NEWS score that resolved during their hospital stay with no intervention or urgent medical review required.

\section{Department of Cardiothoracic Surgery, Cork University Hospital, Cork,} Republic of Ireland 